\title{
ONE-PARAMETER SEMIGROUPS OF ISOMETRIES
}

\author{
BY C. A. BERGER AND L. A. COBURN
}

Communicated by I. M. Singer, March 2, 1970

Let $t \rightarrow V_{t}$ for $t \geqq 0$ be a strongly continuous one-parameter semigroup of isometries on a Hilbert space $H$. The easiest example of such a semigroup which is not unitary is given by considering the Hilbert space $\widetilde{H}=L^{2}(0, \infty)$ consisting of those Lebesgue square-integrable functions on $(-\infty, \infty)$ which are supported on $(0, \infty)$. On $\widetilde{H}$, we consider the (nonunitary) isometries

$$
\left(T_{t} f\right)(x)=f(x-t) .
$$

Recently, the $C^{*}$-algebra $a\left(T_{t}: t \geqq 0\right)$ generated by the semigroup $t \rightarrow T_{t}$ has been studied in detail [2], [3], [4].

In this note, we show that for any strongly continuous one-parameter semigroup of isometries $t \rightarrow V_{t}$ with $V_{t_{0}}$ not unitary for some $t_{0}, \mathbb{Q}\left(V_{t}: t \geqq 0\right)$ is *-isomorphic with $\mathbb{Q}\left(T_{t}: t \geqq 0\right)$. The proof is modelled after the corresponding result for $C^{*}$-algebras generated by a single isometry [1].

The main fact that we use is a generalization due to Cooper [6, p. 142] of the Wold decomposition of a single isometry [5, p. 109]. This generalization states that for $t \rightarrow V_{t}, t \geqq 0$, a strongly continuous one-parameter semigroup of isometries on $H$, there is a Hilbert space $K$ with a strongly continuous one-parameter unitary semigroup $t \rightarrow U_{t}$ on $K$, there is a cardinal $\alpha$, and there is an isometry $U$ from $H$ onto $K \oplus \widetilde{H} \oplus \cdots \oplus \widetilde{H} \oplus \cdots$ where $\widetilde{H}$ occurs with multiplicity $\alpha$, such that

$$
U V_{t} U^{*}=U_{t} \oplus T_{t} \oplus \cdots \oplus T_{t} \oplus \cdots .
$$

The multiplicity $\alpha$ is a unitary invariant which can be read off from the infinitesimal generator of $t \rightarrow V_{t}$ [6, p. 142].

In case $K=\{0\}$, we say that $t \rightarrow V_{t}$ is purely nonunitary [6, p. 136]. For such semigroups, the multiplicity $\alpha$ is the only unitary invariant. A very general way of generating such semigroups is to consider for any measure $d \mu$ which is positive, of bounded variation, and singular with respect to Lebesgue measure on the unit circle $T$, the singular inner functions $\left[5\right.$, p. 66] $\phi_{l}^{\mu}\left(e^{i \theta}\right)$ which are the boundary values of

AMS 1969 subject classifications. Primary 4665, 4750.

Key words and phrases. $C^{*}$-algebras, semigroups of operators. 


$$
\exp \left\{-t \int \frac{e^{i \alpha}+z}{e^{i \alpha}-z} d \mu(\alpha)\right\}, \quad|z|<1 .
$$

It is then easy to check that for $f$ in the usual Hardy space $H^{2}(T)$ $[5$, p. 39],

$$
\left(M_{t}^{\mu} f\right)\left(e^{i \theta}\right)=\phi_{t}^{\mu}\left(e^{i \theta}\right) f\left(e^{i \theta}\right)
$$

defines a strongly continuous one-parameter semigroup of isometries for $t \geqq 0$. The second result of this note shows that $t \rightarrow M_{t}^{\mu}$ is purely nonunitary and characterizes the multiplicity $\alpha(\mu)$ of $t \rightarrow M_{t}^{\mu}$ directly in terms of the measure $\mu$.

TheOREM A. Let $t \rightarrow V_{t}, t \geqq 0$, be a strongly continuous one-parameter semigroup of isometries with $V_{t_{0}}$ nonunitary for some $t_{0}$. Then the $C^{*}$ algebra $Q\left(V_{t}: t \geqq 0\right)$ generated by the $V_{t}$ is ${ }^{*}$-isomorphic with $Q\left(T_{t}: t \geqq 0\right)$.

Proof. Applying the decomposition of Cooper to $t \rightarrow V_{t}$, we see that the problem is reduced to studying

$$
a=a\left(U_{t} \oplus T_{t} \oplus \cdots \oplus T_{t} \oplus \cdots: t \geqq 0\right),
$$

where $T_{t}$ occurs with multiplicity $\alpha \geqq 1$. Now $Q$ is just the normclosure of direct sums of the form

$$
\sum_{j=1}^{n} a_{t_{j}, s_{j}} U_{t_{j}} U_{s_{j}}^{*} \oplus \sum_{j=1}^{n} a_{t_{j}, \varepsilon_{j}} T_{t_{j}} T_{s_{j}}^{*} \oplus \cdots .
$$

The mapping $\Phi$ which sends such a direct sum to

$$
\sum_{j=1}^{n} a_{t_{j}, s_{j}} T_{t_{j}} T_{s_{j}}^{*}
$$

clearly extends to a ${ }^{*}$-homomorphism from $a$ onto $a\left(T_{t}: t \geqq 0\right)$. To check that $\Phi$ is actually a ${ }^{*}$-isomorphism, it suffices to show that

$$
\left\|\sum_{j=1}^{n} a_{t_{j}, s_{j}} U_{t_{j}} U_{s_{j}}^{*}\right\| \leqq\left\|\sum_{j=1}^{n} a_{t_{j}, s_{j}} T_{t_{j}} T_{s_{j}}^{*}\right\| \text {. }
$$

The structure of the algebra $a\left(T_{t}: t \geqq 0\right)$ has been described in [2], [3]. We use the fact that $Q\left(T_{t}: t \geqq 0\right)$ contains a proper closed twosided ideal $\mathbb{C}$ (the commutator ideal) and for $R$ the real line,

$$
\inf _{C \in \mathrm{e}}\left\|\sum_{j=1}^{n} a_{t_{j}, s_{j}} T_{t_{j}} T_{s_{j}}^{*}+C\right\|=\sup _{x \in R}\left|\sum_{j=1}^{n} a_{t_{j}, s_{j}} \exp \left[i\left(t_{j}-s_{j}\right) x\right]\right| .
$$

It follows that it will be enough to show that 


$$
\left\|\sum_{j=1}^{n} a_{t_{j}, s_{j}} U_{t_{j}} U_{s_{j}}^{*}\right\| \leqq \sup _{x \in R}\left|\sum_{j=1}^{n} a_{t_{j, b_{j}}} \exp \left[i\left(t_{j}-s_{j}\right) x\right]\right| .
$$

Now noting that $t \rightarrow U_{t}$ is a strongly continuous semigroup for $t \geqq 0$, we see that $U_{t}$ commutes with $U_{s}^{*}$ and $t \rightarrow U_{t}$ can be extended to a unitary representation of $R$ by defining $U_{-t}=U_{t}^{*}$ for $t \geqq 0$. The desired inequality is obtained by observing that for some selfadjoint (not necessarily bounded) $A$ on $H$,

$$
\left\langle U_{t} f, g\right\rangle=\int_{x \in \sigma(A)} e^{i t x} d\langle E(x) f, g\rangle, \quad t \in(-\infty, \infty),
$$

where $f$ and $g$ are in $H, A$ is the infinitesimal generator for $t \rightarrow U_{t}$, $E(x)$ is the spectral family for $A$, and $\sigma(A)$ is the spectrum of $A$ $(\sigma(A) \subset R)[6$, p. 134]. Hence, using the fact that for $\|f\|=1$

$$
\int_{x \in \sigma(A)} d\langle E(x) f, f\rangle=1
$$

we see that for $\|f\|=1$

$$
\begin{aligned}
\left\|\sum_{j=1}^{n} a_{t_{j, s_{j}}} U_{t_{j}} U_{s_{j}}^{*}\right\|^{2} & =\sum_{j=1}^{n} \sum_{k=1}^{n} \bar{a}_{t_{j, s_{j}} a_{t_{k, e_{k}}}\left\langle U_{s_{j}-t_{j}+t_{k}-s_{k}} f, f\right\rangle} \\
& =\int_{x \in \sigma(A)}\left|\sum_{j=1}^{n} a_{t_{j, s_{j}}} \exp \left[i\left(t_{j}-s_{j}\right) x\right]\right|^{2} d\langle E(x) f, f\rangle
\end{aligned}
$$

and the desired inequality follows.

THEOREM B. The strongly continuous one-parameter semigroup of isometries $t \rightarrow M_{t}^{\mu}$ described above is purely nonunitary and the multiplicity $\alpha(\mu)$ is determined as follows: $\alpha(\mu)=n$ if the support of $\mu$ consists of exactly $n$ points, $\alpha(\mu)=\infty$ otherwise.

Proof. Let us first show that if $w$ is any nonconstant inner function [5, p. 62], then for $f$ in $H^{2}(T)$, the isometry $\left(M_{w} f\right)(z)=w(z) f(z)$ is purely nonunitary. Otherwise, for some $f$ in $H^{2}(T)$ with $\|f\|=1$, $\left\|M_{w}^{* n} f\right\|=1$ for $n=1,2, \cdots$, or equivalently, for $g_{n}$ in $H^{2}(T)$

$$
f=w^{n} g_{n}, \quad n=1,2, \cdots .
$$

Thus, if $w\left(z_{0}\right)=0$ for $\left|z_{0}\right|<1$ then $f$ has a zero of infinite order at $z_{0}$, which is impossible. Thus, $w$ is purely singular and nonconstant so

$$
w(z)=\exp \left\{-\int \frac{e^{i \alpha}+z}{e^{i \alpha}-z} d \nu(\alpha)\right\}
$$

where $\nu$ is a uniquely determined finite positive singular measure on 
$T, 0<\nu(T)<\infty[5$, p. 66]. Equating the singular parts of the functions in $(*)$, we see that for

$$
\begin{aligned}
f_{\text {sing }}(z) & =\exp \left\{-\int \frac{e^{i \alpha}+z}{e^{i \alpha}-z} d \sigma(\alpha)\right\}, \\
\left(g_{n}\right)_{\text {sing }}(z) & =\exp \left\{-\int \frac{e^{i \alpha}+z}{e^{i \alpha}-z} d \tau_{n}(\alpha)\right\}
\end{aligned}
$$

where $\sigma$ and $\tau_{n}$ are finite positive singular measures on $T$, we have $\sigma=n \nu+\tau_{n}$ so $\sigma(T) \geqq n \nu(T)$ for $n=1,2, \cdots$. Since $\sigma(T)<\infty$ and $\nu(T)>0$, we have a contradiction.

We remark further that the defect of $M_{w}$ (the dimension of $\left.\operatorname{kernel}\left(M^{*}\right)\right)$ is finite if and only if $w$ is a finite Blaschke product, and that in this case the defect equals the number of terms in the Blaschke product. We now prove this assertion. Certainly, if $w=\prod_{k=1}^{N} w_{k}$, where the $w_{k}$ are nonconstant inner functions, then $M_{w}=\prod_{k=1}^{N} M_{w_{k}}$. Each of the $M_{w_{k}}$ are purely nonunitary isometries and so have defect at least one. Thus, since

$$
\operatorname{defect}\left(M_{w}\right)=\sum_{k=1}^{N} \operatorname{defect}\left(M_{w_{k}}\right)
$$

(this follows from elementary index-type argument), we have defect $\left(M_{w}\right) \geqq N$. It follows easily that if $w$ has a nonconstant singular part or a Blaschke part with infinitely many zeros, $\operatorname{defect}\left(M_{w}\right)=\infty$. If

$$
w(z)=\lambda \prod_{k=1}^{N}\left(\frac{z-a_{k}}{1-\bar{a}_{k} z}\right)
$$

where $\lambda$ is a constant, $|\lambda|=1$ and $\left|a_{k}\right|<1$, then

$$
\operatorname{defect}\left(M_{w}\right)=\sum_{k=1}^{N} \operatorname{defect}\left(M_{\left(\left(z-a_{k}\right) /\left(1-\bar{a}_{k} z\right)\right)}\right)
$$

and each $M_{\left(\left(z-a_{k}\right) /\left(1-a_{k} z\right)\right)}$ has defect one.

Now by the result of Foias and Nagy [6, p. 142], $\alpha(\mu)$ is just the defect of the isometry obtained by taking the Cayley transform of the infinitesimal generator of the semigroup $t \rightarrow M_{t}^{\mu}$. The infinitesimal generator of the semigroup is $M_{\psi}$, where $\psi$ is the function

$$
\psi(z)=-\int \frac{e^{i \alpha}+z}{e^{i \alpha}-z} d \mu(\alpha), \quad|z|<1 .
$$

The Cayley transform of $M_{\psi}$ is $M_{w}$, where $w=(1+\psi) /(1-\psi)$. Since $\operatorname{Re} \psi(z)<0$, we see that $|w(z)|<1$ for $|z|<1$. Since $\mu$ is singular, 


$$
\operatorname{Lim}_{\rho \rightarrow 1^{-}} \operatorname{Re} \psi\left(\rho e^{i \theta}\right)=0 \text { a.e. }(\theta)
$$

so $w$ is an inner function.

By the foregoing, it suffices to show that $w$ is a finite Blaschke product of $n$ terms if and only if $\operatorname{support}(\mu)$ is a finite set with $n$ points. But if $\operatorname{support}(\mu)$ is finite (with $n$ points) then $w(z)$ is a rational function. The only rational inner functions are finite Blaschke products $[5$, p. 76] so w has the form

$$
w(z)=\lambda \prod_{k=1}^{m}\left(\frac{z-a_{k}}{1-\bar{a}_{k} z}\right)
$$

where $|\lambda|=1$ and $\left|a_{k}\right|<1$. But the zeros of $w(z)$ in the plane are those of

$$
1+\psi(z)=1-\sum_{k=1}^{n} \frac{\exp \left[i \alpha_{k}\right]+z}{\exp \left[i \alpha_{k}\right]-z} t_{k}, \quad t_{k}>0,
$$

and multiplying by $\prod_{k=1}^{n}\left(\exp \left[i \alpha_{k}\right]-z\right)$ we see that $1+\psi(z)$ has exactly $n$ zeros so that $n=m$. Conversely, suppose

$$
w=\lambda \prod_{k=1}^{n}\left(\frac{z-a_{k}}{1-\bar{a}_{k} z}\right), \quad|\lambda|=1 \text {. }
$$

Then

$$
-\int \frac{e^{i \alpha}+z}{e^{i \alpha}-z} d \mu(\alpha)=\psi(z)=\frac{w(z)-1}{1+w(z)}
$$

is a rational function and so has at most finitely many points of $T$ in its natural boundary. But then $\operatorname{support}(\mu)$ contains only those points $[5$, p. 68], and so is finite. This completes the proof.

\section{REFERENCES}

1. L.A. Coburn, The $C^{*}$-algebra generated by an isometry, Bull. Amer. Math. Soc. 73 (1967), 722-726. MR 35 \#4760.

2. L. A. Coburn and R. G. Douglas, Translation operators on the half-line, Proc. Nat. Acad. Sci. U.S.A. 62 (1969), 1010-1013.

3. - On $C^{*}$-algebras of operators on a half-space. I, (to appear).

4. L. A. Coburn, R. G. Douglas, D. G. Schaeffer and I. M. Singer, On $C^{*}$. algebras of operators on a half-space. II: Index theory, (to appear).

5. K. Hoffman, Banach spaces of analytic functions, Prentice-Hall Series in Modern Analysis, Prentice-Hall, Englewood Cliffs, N.J., 1962. MR 24 \#A2844.

6. B. Sz.-Nagy and C. Foiaş, Analyse harmonique des opérateurs de l'espace de Hilbert, Masson, Paris and Akad. Kiado, Budapest, 1967. MR 37 \#778.

Belfer Graduate School of Sciences, Yeshiva University, New York, NEW YORK 10033 
